\title{
REAL-WORLD DATA: EVALUATION OF SENTINEL LYMPH NODE BIOPSY AFTER COMPLETE PATHOLOGICAL RESPONSE OF BREAST CANCER TO NEOADJUVANT CHEMOTHERAPY IN PATIENTS WITH TRIPLE-NEGATIVE AND HER2 POSITIVE BREAST CANCER IN A COHORT FROM RIO GRANDE DO SUL, BRAZIL
}

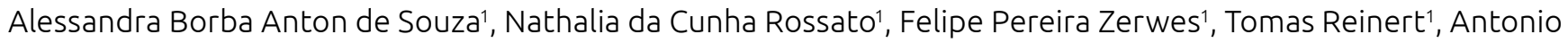
Luiz Frasson ${ }^{1}$

'Pontifícia Universidade Católica do Rio Grande do Sul - Porto Alegre (RS), Brazil.

Introduction: International publications show a high correlation of axillary response and complete pathological response (CPR) of breast cancer to neoadjuvant chemotherapy (NACT) in patients with triple-negative (TN) and HER2 positive (HER2+) tumors. The need for surgery is being questioned when percutaneous breast biopsy after NACT indicates CPR, despite recent presentations demonstrating high rates of false-negative (FN), ranging from 17-39\%. The proper axillary management in patients with CPR of breast cancer is still discussed: is it possible to avoid the axillary evaluation? What is the axillary downstaging rate? Identifying any residual disease to adjust the adjuvant treatment is also a concern. Retrospective studies reveal a rate of positive lymph nodes lower than $2 \%$ in this population when CPR of breast cancer is reached. Objective: To identify the rate of complete axillary response in patients with CPR of breast cancer to NACT in TN and HER2+ tumors. Methods: This is a retrospective cohort study conducted in two health facilities in Southern Brazil. The sample consists of 130 patients who underwent NACT, followed by surgery between January 2016 and December 2018. The patients included were treated in the public health system (Sistema Único de Saúde - SUS) and private health system. Results: Among the 130 patients submitted to NACT, 76 (58\%) had HER2+ and TN immunohistochemical subtypes - luminal HER2+: 23 patients, HER2+ pure: 15, TN: 38. Among these patients, 33 (43\%) reached CPR of breast cancer, of which 9 corresponded to luminal HER2+, 10 to HER2+, and 14 to TN. In patients with CPR of breast cancer, 29 (87.8\%) had no lymph node disease. Out of the 10 HER2+ pure with CPR of breast cancer, 100\% had no lymph node disease, and 8 were positive pre-NACT. Among the $14 \mathrm{TN}$, only 1 patient had 2 positive lymph nodes (2+/10), and she was $\mathrm{cN} 0$ prior to NACT (with negative axillary ultrasound). Among the 5 pre-NACT clinically positive lymph nodes in TN patients (including 1 patient with cN2), all had CPR to NACT (3 axillary dissections and 2 sentinel lymph node biopsies - SLNB). Out of the 9 patients with luminal HER and CPR of breast cancer, 4 had clinically positive lymph nodes before NACT, and 3 remained positive (15\% of conversion). Conclusion: In this study, CPR of breast cancer was highly correlated with negative axillary evaluation after NACT (87.8\%), mainly in the TN and HER2+ pure subtypes (98\%), even if the lymph node was clinically positive before NACT, with $100 \%$ of conversion of HER $2+$ pure cases. SUS patients used trastuzumab as the single drug targeting anti HER2. These data agree with those found in the literature, despite the small sample. Larger studies are necessary, as around 70\% of our population depend on SUS. With more published data, considering the performance of SLNB in HER2+ pure and TN patients submitted to NACT could become a common practice, reducing morbidity. The safety of this practice in the luminal HER+ subtype remains unclear. 